\title{
Multidimensionality of assessment in the Common European Framework of Reference for languages
}

(CEFR)

\author{
Enrica Piccardo \\ enrica.piccardo@utoronto.ca \\ OISE, UNIVERSITY OF TORONTO
}

\author{
Ce n'est pas le doute, \\ c'est la certitude qui rend fou \\ F. Nietzsche, Ecce Homo
}

\begin{abstract}
This article intends to discuss complexity of assessment by presenting its several layers and dimensions as they are conceptualized in the Common European Framework of Reference for languages (CEFR) and to show how the CEFR advocates an inclusive vision of assessment able to integrate several perspectives. After presenting the CEFR perspective of the nature and role of assessment, the article investigates some challenges practitioners are facing and their needs as to the assessment process. It also aims at casting light on the actual and potential impact of the CEFR on assessment cultures in different contexts. The data presented in this article, collected within the ECEP (Encouraging the Culture of Evaluation among Professionals) project of the Council of Europe and within its extension in the Canadian context, will help to understand why the CEFR can be seen as a relevant awareness-raising tool in the domain of assessment and beyond.
\end{abstract}

Key words: CEFR, assessment, complexity, reflective practice, language proficiency

\section{Résumé}

Cette contribution discute de la complexité de l'évaluation en présentant ses différents niveaux et dimensions selon la vision du Cadre européen commun de référence pour les langues (CECR). La perspective adoptée par le CECR est présentée, qui prône une vision inclusive de l'évaluation avec des implications différentes. Cette contribution questionne certains des défis auxquels les praticiens sont confrontés ainsi que les besoins qu'ils éprouvent dans le domaine de l'évaluation. Elle vise aussi à 
clarifier l'impact, réel et potentiel, du CECR sur les cultures d'évaluation propres à des contextes différents. Pour ce faire, une partie des données collectées lors du projet ECEP (Encourager la culture de l'évaluation chez les professionnels) du Conseil de l'Europe et de son extension au contexte canadien sont présentées. Ces données montrent les raisons pour lesquelles le CECR peut être vu comme un outil susceptible d'aider les praticiens à prendre conscience de la complexité de l'évaluation et des ses implications.

Mots-clés : CECR, évaluation, complexité, pratique réflexive, compétence langagière

\section{Introduction}

Research and reflection on assessment in general and in second languages in particular have informed a large number of publications and textbooks over the last two decades and have shown how many layers and implications this crucial phase of the teaching process possesses, and what impact it can have on effective learning (Bachman, 1990; Cumming and Berwick, 1996; Kunnan, 2000; Cumming and Laurier, 2007).

What can be generally observed is that research necessarily tends to focus on specific aspects or layers of assessment - usually very few at a time; for instance (and randomly chosen): validity and practicality, scope and target group, measurement and feedback modalities. This is quite logical, especially if we consider that each of these aspects requires in-depth and targeted investigation and may play a fundamental role in the process. Less attention has been paid to the connections between these different aspects as well as their possible synergies or conflicts and even less to the practical implications that all this has in the everyday Second Language Education (SLE) praxis (Bachman and Palmer, 2010)

The vision introduced in Europe by the Common European Framework of Reference for Languages (CEFR, 2001), aims - as is already stressed by the subtitle "Learning, teaching, assessment" - to give reflection on assessment, the implications of assessment and above all its multidimensionality in the professional discourse, i.e. to treat it consistently within the dynamics of learning/teaching and not as a separate entity (Little, 2006; North, 2009; Gerber, 2008).

The following paragraphs will discuss the multidimensionality of assessment as well as its reasons and implications, on the basis of the vision introduced by the CEFR. We will then extend our reflection to show how in different European contexts (and beyond Europe) similarities can be observed when it comes to issues connected with assessment and to explain potential impacts of the new vision introduced by the CEFR on the learning/teaching process. 
For discussing this aspect, we will draw on some of the ECEP (Encouraging the Culture of Evaluation among Professionals) project data. ECEP is an international research and development project, which we coordinated, funded by the Council of Europe within the 4-year program "Empowering Language Professional" of the European Centre for Modern Languages (ECML) located in Graz, Austria (ecep.ecml.at). Finally, we will address the inevitable limits of the CEFR in terms of providing a comprehensive and ready-to-use solution for assessment in SLE.

\section{Fundamental concepts and main uses of assessment}

When it comes to assessment in the CEFR, which is especially dealt with in Chapter 9, the three fundamental concepts of validity, reliability and feasibility are presented as constituting the underlying foundation of every discourse in this area. Whereas validity is indicated to be a central concern of the CEFR, reliability is partially reconsidered and enlarged: rather than focusing mainly on the rank ordering of replicated measurement outcomes, it stresses the importance of the accuracy of decisions made relative to a standard - for example, assignment to a CEFR level (p. 177). Finally, feasibility is especially mentioned as a fundamental issue due to external constraints and limits under which assessors operate and in relation to the need to be selective with such a comprehensive tool as the CEFR.

But over and above these three fundamental categories, the CEFR puts the two key questions it is concerned with: "What is assessed?" and "How is performance interpreted?" (p. 178), thus opening the second layer; that is, how the CEFR can be used.

The answer provided to this question by the CEFR itself is that the CEFR can be used to specify the content of tests (what is assessed), to formulate criteria capable of determining the attainment of objectives (how to interpret the performance), and to describe levels of proficiency which eventually allow comparison between institutions and, at the international level, between examinations, including for different languages (how to compare).

\section{Two poles: The performance and behind}

The first two layers mentioned, underlying principles and possible uses of the CEFR, can be linked to what the CEFR defines as a 'horizontal' dimension, i.e. the set of descriptive categories introduced in Chapters 4 and 5 map out a 'horizontal dimension' made up of parameters of communicative activity and communicative language competence (p. 16).

But for the CEFR this horizontal dimension, which is made up of these two complementary poles (Hulstijn, 2011, p. 239) is complemented by a 'vertical' dimension represented by an ascending series of common reference levels for 
describing learner proficiency (p. 16) outlined in Chapter 3 (Little, 2006).

The pole represented by the communicative language competence refers to the competences that a language user draws upon and puts into action in order to accomplish a task. For the CEFR, the difference between competence and performance is not an ideological one, but rather a functional one, where assessment focuses mainly on collecting data produced through different communicative activities able to provide evidence of underlying competences.

The movement from the singular "competence", seen by Chomsky (1965) as an abstract knowledge of the language, as the ability to produce and to understand a theoretically infinite number of sentences - even unknown ones to a whole range of different, specific, "competences" classified into two categories, general and linguistic, according to the descriptive scheme of the CEFR, is a movement from abstract to concrete, from ideological to functional, from theoretical to applied.

Chapter 4 of the CEFR focuses on descriptors of communicative activities. In the action-oriented perspective, a thorough description of what actually happens during task performance, be it in reception or in production/interaction, is essential; this description provides support in describing tasks, which allows elaborating targeted and focused tests, is important for providing a reliable and clear snapshot of what is expected by the learner.

A snapshot is a way of describing and recording what the learner can do at a precise moment. Nevertheless, in order for assessment to be effective, i.e. to go beyond description and towards interpretation, another support is necessary, which can help practitioners to elaborate criteria and transform impressions into well-grounded judgments.

Descriptors of competences included in Chapter 5 of the CEFR provide practitioners with a good basis for describing and categorizing what can be inferred through the performance, eventually for designing a performance profile.

With this choice of underlying complementarity between communicative activities and communicative competences, the CEFR intends to overcome the big theoretical dichotomy between use and ability, between what learners can do, i.e. the specification of task performance, and what the underlying abilities of those same learner are, i.e. the construct behind, what is not directly observable and incorporate the more holistic vision of communicative competence as well as of language teaching and testing (Hymes, 1971, Savignon, 1997; Canale and Swain, 1980).

All the data collected during all different forms of testing need appropriate ways of presentation, and the choice of these ways, as we will see later, is not irrelevant when it comes to see assessment as a way for interpreting data and providing feedback. 


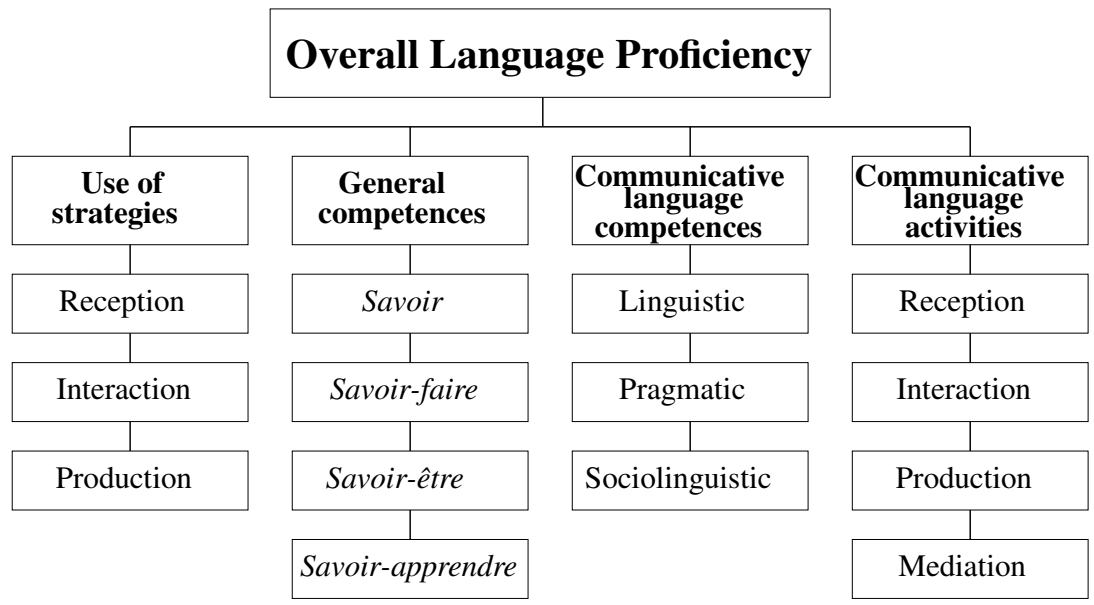

\section{FiguRE 1}

CEFR: A descriptive outline (Piccardo, Berchoud, Cignatta, Mentz and Pamula, 2011, p. 55)

\section{The descriptive scheme of the CEFR}

The communicative competence of individuals is made up of very diverse components and the CEFR classifies them in the descriptive scheme ${ }^{1}$ shown in Figure 1 , which is a simplified graphical representation.

The main distinction remains the one mentioned before, going from communicative language activities to underlying competences - both general and communicative ones. The complex nature of competences is stressed by the distinction between general competences and communicative language competences. It is intuitively clear that, in the case of language learning, linguistic, pragmatic and sociolinguistic competences play a central role, but it is less generally admitted that more general competences play a fundamental role too, especially the existential competence (savoir-être) and the ability to learn (savoir-apprendre). While knowledge (savoir) and know-how (savoir-faire) seem to replicate the distinction between abstract knowledge and implementation of this knowledge, the other two general competences reach a deeper level of the individual, touching the area of personality and emotions on one side and the metacognitive area on the other.

\footnotetext{
${ }^{1}$ The CEFR states "chapters 4 to 7 elaborate a descriptive scheme, which tries to conceptualize language use, competences and the process of teaching and learning in a practical way which will help partners to operationalize the communicative language ability we wish to promote" (p. 182).
} 
Finally, the use of strategies contributes to link what I referred to in the above section as the two poles and they may also be seen as a way for linking the vertical and the horizontal dimensions of language use and language proficiency. Learners use strategies in several senses: to activate competences in order to perform tasks which require communicative activities and to learn from their performance which strategies are the most effective and most contribute to increase their different competences. Thus the movement goes from the pole of the task performance to the pole of the different competences and vice versa but it also goes from the horizontal dimension of language use to the vertical definition of language proficiency organized into ascending levels.

\section{Assessment tools and resources}

As the CEFR advocates for itself the role of a tool based on recent linguistic and pedagogic theories, which aims at informing SLE praxis, resources are provided and organized in a way that allows practitioners to come to terms with its wealth of underpinning concepts. Nevertheless, this process is not always straightforward. Moreover, in order to be effective, the assessment process needs to be as accurate and as targeted as possible. And for this reason several assessment tools and resources need to be at the practitioners' disposal. Descriptors, grids, checklists, tables and scales all constitute possible ways of organizing data which are functional to the goal and target group of the assessment action. Those included in the CEFR or in other strictly connected documents (Council of Europe, 2009; see also www.coe.int/t/DG4/Portfolio/? $\mathrm{L}=\mathrm{E} \& \mathrm{M}=/ \mathrm{main} \_$pages/portfolios.html for different models of validated European Language Portfolios). constitute both a databank from which practitioners can select (and if necessary customize/adapt) and possible models for personalized tools.

The fundamental distinction is linked to both the two dimensions indicated earlier, the horizontal and the vertical one, and the two poles, language activities and communicative language competence. A grid such as the selfassessment grid (CEFR, 2011, Table 2, pp. 26-27) gives a snapshot of the learners' proficiency level in the different communicative activities. A scale such as the global scale (CEFR, Table 1, p. 24) not only is a snapshot, a more distant and less detailed one, but also a tool for giving a sense of where the learner is in the progression. In turn, these snapshots can be analyzed and detailed by specifying qualitative aspects of the performance such as in Table 3 of the CEFR (pp. 28-29). In order to support practitioners in their construction of targeted grids, the descriptors provided by Chapter 4 (communicative activities) and Chapter 5 (competences) are complementary and cater for both poles of the horizontal dimension, favouring metacognitive reflection and supporting a constant movement between aspects of the performance and what is implied 
in this performance, what makes this performance the way it is. At the same time they pave the way to the vertical dimension as they provide transparency thus supporting progress through the levels. An action-oriented assessment is enriched by this dynamic comparison between observable data and implied competences, as the process clarifies strengths and weaknesses of the learners thus orienting pedagogic actions and/or autonomous learning.

Descriptors of strategies, also provided by the CEFR (mainly in Chapter 4) are therefore able to scaffold this entire process and make it even more transparent and effective.

Assessment tools can also be seen (and used) as a kind of blueprint of the learning journey. This is the case of checklists, another way of presenting descriptors and of guiding and "signposting" the learning journey. But it is also the case of empty grids, such as those presented in the language passport, ${ }^{2}$ which need to be filled in little by little in order to visualize the proficiency of a learner at a precise moment in time.

There is no doubt that the format of presentation and the type of tool chosen not only make a great difference as each allows sending different messages to the learner and focusing on different aspects, but also the very fact of having to choose the way of describing and presenting criteria, levels and specifications adds another layer to the process of assessing, i.e. the need to answer the question "How to present?".

\section{Assessment in context}

Language teachers, as every professional, do not live in a vacuum, but have to cope with specific situations, clearly defined contexts and a set of constraints (Tudor, 2001). Assessment is usually one of the most institution-driven areas of their professional activities and what institutions mainly require from assessment is for practitioners to be able to define a level of proficiency, to provide a score, a grade, and to respect the norm, usually within a clearly defined culture of evaluation.

The need for practitioners to constantly deal with both the vision of assessment proposed by the CEFR and their own specific contexts and constraints was one of the reasons at the basis of the Encouraging the culture of evaluation project (ECEP) financed by the Council of Europe. ${ }^{3}$ This four-year international project aimed at investigating the attitude of language teachers towards

\footnotetext{
${ }^{2}$ The language passport is one of the three components of the European Language Portfolio; see the dedicated website www.coe.int/t/dg4/education/elp/. The Language passport can be downloaded from www.coe.int/t/DG4/Portfolio/documents/Pass_2spr.pdf.

${ }^{3}$ Encouraging the culture of evaluation among professionals: The case of language teachers (ECEP) (http://ecep.ecml.at/) is a project of the European Centre for Modern Languages (ECML), a Council of Europe institution promoting excellence in language
} 
assessment, and the impact of the CEFR on these attitudes as well as on their assessment practices. The aim of the project was to create a tool able to assist practitioners in the paradigm shift advocated by the CEFR.

According to the data collected, teachers from four different countries found it very challenging to cope with institutional constraints while implementing the CEFR, in particular to find a balance between providing both detailed descriptors and a synthetic appreciation of proficiency levels, to effectively link grading and assessing and to deal with unbalanced profiles.

The CEFR claims to be sufficiently universal and non-dogmatic for being used in potentially every context. The application/adaptation of the CEFR well beyond the European borders shows that this claim is fundamentally true but it also confirms the same difficulties as reported above (Coste, 2007).

Practitioners are, in fact, requested to add another layer to their reflection on assessment, namely taking into consideration both specific contextual aspects and universally oriented tools and resources.

We will not present in detail the data collected during the ECEP project, which were used to inform the final publication, as we have already presented them from two different perspectives in two publications (Piccardo, 2011a, 2011b), but we will present a synthesis in order to help the reader situate the project and the perspective adopted in this article.

We will then concentrate on the concepts presented by the CEFR in its ninth chapter dedicated to assessment in order to exemplify both issues related by practitioners and choices adopted during the ECEP project.

\section{A synthesis of the ECEP project: Some data for reflection}

The team that worked at the ECEP project was composed by a core group ( 5 researchers from 4 countries - France, Italy, Poland and Germany) to which a second supporting network was added, composed by eight experts from eight other European countries. Another panel of experts coming from other European countries were then consulted to give feedback on the materials produced. The core team plus members of the supporting network piloted the different materials produced.

During the first phase of the project the core team formulated four hypotheses linked to the CEFR (partial knowledge of the CEFR by practitioners, usually limited to some descriptors; difficulty in integrating the CEFR into everyday practice; lack of targeted training related to the CEFR; lack of support and resources in the implementation of the CEFR). They then tested these hypotheses during data collection which took place in two phases: an exploratory phase done with the help of a survey followed by interviews and a more in-

education in its 34 member states (www.ecml.at/). 
depth phase conducted through a series of focus groups specifically targeting the assessment dimension, assessment practices and impact of the CEFR on assessment and pedagogical choices.

Assessment is a notoriously complex dimension of language teaching. In a sense, the more practitioners reflect upon the different aspects and implications of assessment the more they need to reflect. In fact, often what they find represents both a possible solution to some issues and the beginning of a new issue, for considering new implications and consequences.

This feeling was confirmed during data collection for the ECEP project. The focus groups in different contexts revealed how practitioners found themselves at some phase in this process, how differently various implications were perceived and different strategies implemented.

From the data we also noticed that the wealth of perspectives that the CEFR presents both in assessment and in language learning and teaching in general, the multidimensionality of assessment we discussed above, which reveals the complexity of the language learning process, resulted in opening up novel considerations for the role of assessment among practitioners.

In a nutshell, we observed some significant changes of perspective among practitioners who had been exposed to the CEFR and had tried to implement it. The first was a much deeper awareness of the different components of the assessment process and of the fact that such complexity was somehow made visible by the CEFR. A second important aspect was the understanding of the distinction between the assessment process and the grading process, which was far from automatic for most of the participants in the research. Also the possibility of assessing and grading different elements of the performance and the underlying competences appeared as a new possibility, seen by most of the participants as very positive and as rather worrying by some of them. The new role of the oral components, especially interaction, was also generally acknowledged and so was a sense of freedom in relation to the curriculum. Finally a new, positive, vision of errors and of their role was one of the most striking aspects observed.

On the whole, despite some sense of anxiety and insecurity, teachers perceived the CEFR as an asset and a powerful tool for improving their practice. Nevertheless, most of them felt a big gap between the tool and the possibility of effective implementation.

Generally speaking we could classify the fundamental needs of teachers in the assessment domain in three categories:

- The need for a comprehensive tool

- The need for examples

- The need for being selective 
The first need can be covered by the CEFR itself. All participants in the study agreed upon seeing in the CEFR a sufficiently comprehensive tool to lay the foundations for effective assessing and also for language teaching. Some even thought that it was "too comprehensive" in the sense that it aimed at covering so many different aspects, but this was generally seen as still quite positive.

Practitioners, on the contrary, saw the second need as more problematic. At the moment of data collection (2008-2009) not many examples were provided to the teachers. Even though we noticed differences between contexts, the gap between the CEFR and its possible implementation in everyday practice was seen as a major problem, above all due to lack of specific contextualized examples. The situation improved dramatically when teams of practitioners worked together to customize ideas of the CEFR to their own context and the shared efforts of customization brought an impressive improvement in reflection and awareness. It is well documented that reflection and discussion highly impact teachers beliefs and practices; nevertheless we could also witness how practitioners had to face a double challenge: making sense of the conceptual density of the CEFR and understanding the different layers and implications of assessment presented in this paper and at the same time figuring out how all this could be made compatible with their own context, their institutional constraints and the culture of evaluation they were dealing with. This confirmed us of the need for a tool to support understanding and reflection of the CEFR and resources and guidance able to scaffold the contextualisation process.

Finally, the third need concerns the ability of practitioners to be selective, to be able to find their own way through the labyrinth, not a random choice but rather a targeted decision-making process. This is perhaps the most challenging need as it requires, on top of a solid professional competence and knowledge, in-depth work on awareness raising and on confidence building as well as the will to take risks and to enter into a recurrent action-research process. This consideration paved the way to our four-year ECEP project and to the shaping of the final product Pathways through assessing, learning and teaching in the CEFR (Piccardo et al., 2011).

\section{From micro to macro categories}

The data collected during the project did more than confirm the initial hypotheses. They provided a solid base for structuring the final two-fold tool represented by the Pathways publication, which includes a guide and a toolkit to help grasp the complexity of the CEFR and see its applicability in the different contexts. In this material, all the main concepts of the CEFR underwent a process of analysis and explanation in order to help professionals understand them and to see their interconnected nature and their potential for their own practice. 
Assessment constituted the central focus of the ECEP project, so the aim was not only to help practitioners become familiar with the different concepts of the CEFR but also, above all, to see the links between these different concepts and the assessment process. We will, therefore, elaborate the multidimensional nature of assessment in the CEFR by showing how some of its most difficult sections concerning assessment were brought to the practitioners through the guide and the toolkit, which compose Pathways.

Not only does the CEFR present and discuss fundamental principles of assessment, provide descriptors and classify the various elements that need to be assessed, and cater for both the horizontal and the vertical dimensions of the learning process, but it also multiplies the types of assessment by classifying them into thirteen pairs, which are presented hereafter, each pair being along a continuum.

The choice of this classification is a very innovative one, as it allows for systematization and rationalization of the different aspects and perspectives that are intrinsic to assessment. The presentation along several continua stresses a non-dogmatic vision of the assessment process, which is not in black-and-white terms but which allows for - but also requires - a great deal of decision-making from practitioners: informed, targeted and contextualised decision-making.

After all the layers discussed above though, practitioners may feel rather puzzled and perceive the assessment pairs as another, additional, burden when it comes to assessment and the related decision-making process. This is by no means the case as the fundamental concepts and implications discussed above are all embedded in the assessment pairs presented by the CEFR.

Following the preparatory research to the ECEP project, we collected data showing how consistent this feeling is among practitioners faced with the CEFR and new institutional constraints in assessment. In particular, two important conclusions could be drawn from the data: a shared sense of too many aspects to cope with in case practitioners aim at an effective implementation of the CEFR and a sense of disorientation when faced with the thirteen different assessment pairs.

This helped us in the analysis of the pairs and of the underlying and embedded concepts. We envisaged the following four macrocategories as guiding threads connecting groups of pairs (Piccardo et al., 2011, pp. 47-50): ${ }^{4}$

- Distinguishing competence and action

- Assessment: a question of timing?

- The issue of objectivity in assessment

- Assessing: reasons, actors, modalities

\footnotetext{
${ }^{4} \mathrm{~A}$ thorough and detailed explanation of this classification is provided in Piccardo et al. (2011, pp. 34-38).
} 
The difference between competence(s) and action concerns mainly the following pairs:

- Achievement assessment

- Direct assessment

- Performance assessment
- Proficiency assessment

- Indirect assessment

- Knowledge assessment

Do we need to focus on what we can observe directly, thus drawing conclusions on the level of proficiency? Or rather pay attention to the performance mainly to deduce - and assess - underlying competences, knowledge and achievement?

To what extent are these two perspectives complementary or opposite and separable?

Proficiency assessment can be done through performance assessment, that is, analyses of competences put to use. Learners' achievement is linked to what they know, what they have acquired over a certain period of time. Proficiency involves the application of knowledge to real world tasks. Performance appears from an external view, whereas relevant knowledge remains internal (Piccardo et al., 2011).

The second group of pairs is focused mainly on the issue of timing: a distinction made in the CEFR is the one between using assessment to classify learners either in relation to one another - and, if necessary, in relation to a norm and a precise spot in time - or to judge learners according to their own abilities, regardless of their peers, in respect to a developmental process over a period of time.

- Norm-referencing (NR)

- Mastery learning CR

- Continuous assessment
- Criterion-referencing (CR)

- Continuum CR

- Fixed point assessment

Do we need to consider the performance in relation to a norm, in terms of cut-off points that allow us to discriminate between those who can and those who cannot perform as expected, between some learners and some others? Or do we need to stress the development, the progression in language acquisition and proficiency focusing on some criteria and drawing a map of the learner in relation to them?

The third, and certainly pivotal, question, the issue of objectivity, is particularly evident in the following pairs:

- Subjective assessment

- Impression

- Holistic assessment

- Series assessment
- Objective assessment

- Guided judgment

- Analytic assessment

- Category 
Even though objectivity remains a central issue and a very tough oneor maybe exactly for this same reason - the CEFR prefers talking of objectively scored assessment, thus showing how the way towards objectivity is never coming to an end point but rather remains a work in progress. Nevertheless, beyond this precision, the CEFR underlines the main difference between a more subjective, impression based, synthetic and global assessment and a more objective one, guided by criteria or categories.

Finally, the following pairs compose a more heterogeneous category, which deals with reasons, modalities and actors:

- Formative assessment

- Checking rating

- Assessment by others
- Summative assessment

- Performance rating

- Self-assessment

This category adds some extra questions to the fundamental ones included in the CEFR, which were presented at the beginning of the contribution, i.e. what we assess, how we interpret the performance, and how we compare. Such questions are fundamentally: Why do we assess? To do what? Who is assessing? How? By what means and tools? They complete the picture and enlarge the perspective showing how assessment implies choices, even collective choices, and clear goals and paths.

It is evident, and even intuitive, that there is quite a bit of overlapping between the pairs attributed to each macrocategory, in fact aspects like for instance the issue of objectivity concern all categories and are spread all over the pairs. It would be impossible to subdivide pairs and categories in watertight compartments, it would also be incoherent with the complex approach that the CEFR has chosen for dealing with assessment.

Nevertheless the choice of threads capable of clarifying fundamental concepts of assessment and of linking different pairs to the core reflection of the CEFR as far as the language learning process is concerned is potentially able to simplify diversity while maintaining complexity as an asset ${ }^{5}$ This is very much on the same line of thought which informs the CEFR, where overall language proficiency is detailed in a descriptive scheme, which includes general and linguistic competences, communicative activities and strategies.

This grouping was used in Pathways as a sort of guiding map to help practitioners understand the 13 pairs, to link them to the other layers of the assessment process and to see in what way all of these distinctions would inform and improve their own practice.

\footnotetext{
${ }^{5}$ This type of categorization was presented and shared during the central workshop of the ECEP project with experts from over 30 countries of the Council of Europe and later piloted during several teacher education sessions in different countries.
} 


\section{Issues inside and outside Europe/in Europe and beyond}

As its name indicates the CEFR is a European document, produced with extensive co-operation and consultation, and different European contexts and pedagogic traditions have laid the foundations to it. It was with the aim of increasing comparability, transparency and transferability that this tool was conceived even though nearly immediately it was clear that it would go well beyond this first goal and become a potentially powerful tool for innovation in the whole domain of language learning and teaching. ${ }^{6}$

Both the original purpose of the CEFR and its extended one opened up another perspective, which has recently become more and more visible: the transferability of the CEFR to non-European contexts (Piccardo, Germain-Rutherford and Clément, 2011). This potential is not to be intended as a mere top down application of an institutional document as this would be contrary to the fundamental vision underlying it; it is, on the contrary, the reflection that this tool is fostering among practitioners and researchers that is proving very interesting. The conceptual categories presented by the CEFR as well as the different assessment tools proposed - such as grids, scales and checklists but, above all, its principles and the inspiring philosophy are revealing their potential for innovation in the domain of language teaching.

As the ECEP project investigated issues related to assessment in general and in relation to the implementation of the CEFR, it was interesting to see if other, non-European, contexts where practitioners were not yet familiar with the CEFR witnessed the same, or similar, issues as far as assessment was concerned and therefore to measure the potential of the CEFR in such contexts. In a recent case study we conducted in the Canadian context Mison and Jang, 2011) teachers expressed the need for transparency and consistency in assessment and indicated that this could be attained mainly through the use of clear descriptors, examples and teamwork. An effective assessment was for them one which would be able to inform students, care for different abilities and save time. In the actual situation, the Canadian teachers felt that assessment of the oral component was too weak especially at lower levels, perceived formative assessment as ambiguous and assessment in general as a final obligation, an exterior process. Finally they were rather worried about the value of peerassessment even if they expressed curiosity and interest.

These statements echoed several of the European teachers' concerns showing how some of the central issues linked to assessment are naturally perceived by practitioners even without any contact with the CEFR or any familiarity with other theoretical studies. But what is particularly interesting to notice is how the contact with the CEFR brings practitioners' reflection further by helping

\footnotetext{
${ }^{6}$ See Coste (2007) for a discussion on this issue.
} 
problematize it. Even though by no means all the questions of the interviewed European teachers found a solution - on the contrary some extra questions were added - the reflection tended to move to another level, a higher one, where each context was not forgotten, but rather reconsidered in the light of more general questions. One of the most striking examples of this is the reflection about competences where teachers sounded proud of their new awareness and capacity of assessing separate aspects, thus being much more effective in analyzing data and in introducing targeted actions and teaching strategies. Another very interesting aspect was the new consideration that teachers showed of tasks and of the role of tasks in the learning process in general and in assessment in particular. Finally, transparency was also seen from a different perspective after the implementation of the principles of the CEFR, including the role of self and peer assessment and their advantages and disadvantages.

Even though our Canadian study is still at an initial phase, we consider it as an interesting starting point to question issues, doubts and beliefs that practitioners have in the assessment domain and to compare it with other contexts where the impact of the CEFR is clear and measurable. This is especially true as the CEFR is being taken more and more into consideration in the Canadian reality and is influencing the reshaping of several provincial curricula. ${ }^{7}$

\section{Complexity: Issue or solution?}

Assessing is obviously not the final phase of the teaching/learning process. On the contrary, it can be considered as the driving force of the entire process. But this just in the case assessment is the result of a well-conceived process, it is conducted in a transparent manner with a scientific attitude consisting in collecting and interpreting data for measuring previously determined and targeted learning objectives, and it is user-friendly and able to provide useful feedback.

Such a demanding process cannot be content with easy and straightforward solutions or uniform replies. Besides, the constant need for balance between transparency and practicality adds a huge challenge to the endeavour, as practitioners may feel that they constantly have to choose between what is right and what is possible. Nevertheless, acting on the assessment process is potentially able to impact the whole language teaching/learning process, therefore to foster and also to scaffold innovation.

The CEFR is a complex document; its aim is not that of providing a ready-

\footnotetext{
${ }^{7}$ Several provinces have introduced new curricula for French second language that are more or less openly linked to the CEFR. For instance, the British Columbia curriculum is advocating an explicit link to the CEFR, whereas the new Ontario curriculum does not state that link explicitly but the influence of the CEFR is clearly visible.
} 
made, universal solution to the issues related to assessment. It provides tools and a lot of resources and also general examples (even if all this is not always clearly organized or easy to find) but this wealth is like the painter's palette or the rough material an artisan has access to. It is in itself completely inconsequential and can produce artwork and very refined handcrafts or on the contrary remain totally unexploited. The approach to the CEFR needs, therefore, to be an open one, an approach to complexity (Gwerder, 2008; Piccardo 2010a, 2010b).

In complex systems, not only are all the elements interconnected but also each element is able to influence all the others. Considering the language teaching/learning process as a system allows practitioners to step out of the dichotomies of right or wrong, effective or non-effective, fair or unfair vision of their professional practice in general - and of assessment in particularand to enter in a paradigm of complexity. Once this paradigm shift has been done, practitioners start reasoning in terms of choices and of consequences of these same choices instead of looking for more or less correct solutions. This, in turn, can foster an awareness-building process and eventually improve the whole system.

This awareness of complexity may indeed prove the turning point of a real professional improvement. It can be the precondition of targeted choices and as choices need to be context related, the whole assessment process becomes more effective because more targeted.

The data collected for the ECEP project clearly showed that the CEFR is potentially able to set up what would be defined as a virtuous circle into motion provided that practitioners are supported in their journey towards accepting complexity, openness to risk-taking and sense of freedom in approaching and implementing the CEFR in their practice.

"Encouraging the culture of evaluation among professionals: The case of language teachers", the title we chose for the project, means supporting practitioners in their journey of discovery. Assessment is multidimensional, practitioners should be encouraged in the process of becoming aware of such multidimensionality and of seeing the complexity of their profession as a challenge but also as an asset.

\section{References}

Bachman, L. 1990. Fundamental considerations in language testing. Oxford: Oxford University Press.

Bachman, L. and A. Palmer. 2010. Language assessment practice: Developing language assessments and justifying their use in the real world. Oxford: Oxford University Press. 
Canale, M. and M. Swain. 1980. Theoretical bases of communicative approaches to second language teaching and testing. Applied Linguistics, 1, pp. 1-47.

Chomsky, N. 1965. Aspects of the theory of syntax. Cambridge, MA: MIT Press.

Coste, D. 2007. Contextualising uses of the Common European Framework of Reference for Languages. In The Common European Framework of Reference for Languages (CEFR) and the development of language policies: Challenges and responsibilities, pp. 38-47. Strasbourg: Language Policy Division. Available at: www.coe. int/t/dg4/linguistic/Source/SourceForum07/ForumFeb07_\%20Report_EN.doc.

Council of Europe. 2001. Common European Framework of Reference for Languages: Learning, teaching, assessment. Strasbourg: Council of Europe Publishing/Cambridge: Cambridge University Press. Available at: www.coe.int/t/dg4/linguistic/ Source/ManualRevision-proofread-FINAL_en.pdf.

Council of Europe. 2009. Relating language examinations to the Common European Framework of Reference for Languages: Learning, teaching, assessment $(C E F R)-A$ manual. Strasbourg: Language Policy Division. Available at: www.coe.int/t/dg4/linguistic/Source/Manual\%20-\%20Extra\%20Material\%20-\%20proofread \%20-\%20FINAL.doc.

Cumming, A. and R. Berwick (eds.). 1996. Validation in Language Testing. Clevedon, UK: Multilingual Matters.

Cumming, A. and M. Laurier (eds.). 2007. Canadian Modern Language Review, 64,1. Thematic issue: Language assessment.

Gerber, B. 2008. Le CECR, un outil pour évoluer. Babylonia, 2, pp. 64-65.

Gwerder, S. 2008. Le CECR: un instrument utile mais qu'il faut maitriser pour l'appliquer. Babylonia, 2, p. 65.

Hulstijn, J.H. 2011. Language proficiency in native and nonnative speakers: An agenda for research and suggestions for second-language assessment. Language Assessment Quarterly, 8, pp. 229-249.

Hymes, D.H. 1971. On communicative competence. Philadelphia: University of Pennsylvania Press.

Kunnan, A.J. (ed.). 2000. Fairness and validation in language assessment. Cambridge: Cambridge University Press.

Little, D. 2006. The Common European Framework of Reference for Languages: Content, purpose, origin, reception and impact. Language Teaching, 39, pp. 167-190.

Mison, S. and I.C. Jang. 2011. Canadian FSL teachers' assessment practices and needs: Implications for the adoption of the CEFR in a Canadian context. Synergies Europe 6. Krakow: GERFLINT, pp. 99-108.

North, B. 2009. The relevance of the CEFR to teacher training. Babylonia, 2, pp. 55-57.

Piccardo, E. 2010a. From communicative to action-oriented: New perspectives for a new millennium. CONTACT TESL Ontario, 36.2, pp. 20-35. 
Piccardo, E. 2010b. L'enseignant, un stratège de la complexité: quelles perspectives pour la formation? In G. Baillat, D. Niclot and D. Ulma (eds.), La formation des enseignants en Europe : approche comparative, pp. 79-98. Brussels: de Boeck.

Piccardo, E. 2011a. "Assessment recollected in tranquillity": The ECEP project and the key concepts of the CEFR. Paper presented at Association of Language Testers in Europe (ALTE) conference, Krakow, Poland.

Piccardo, E. 2011b. Du CECR au développement professionnel: pour une démarche stratégique. Tendances en didactiques des langues secondes et en formation des enseignant(e)s. Revue canadienne de linguistique appliquée, 15.2, pp. 20-52. Available at: ojs.vre.upei.ca/index.php/cjal/article/view/1026.

Piccardo E., M. Berchoud, T. Cignatta, O. Mentz and M. Pamula. 2011. Pathways through assessing, learning and teaching in the CEFR (+ CDrom and website). Strasbourg: Council of Europe.

Piccardo, E., A. Germain-Rutherford and R. Clément (eds.). 2011. Adopter ou adapter : le Cadre européen commun de référence est-il seulement européen ? Synergies Europe 6. Cracow: GERFLINT.

Savignon, S.J. 1997. Communicative competence: Theory and classroom practice. 2nd ed. New York: McGraw-Hill.

Tudor, I. 2001. The dynamics of the language classroom. Cambridge: Cambridge University Press. 